\title{
A Novel Analytical Solution Method for Constraint Forces of the Kinematic Pair and Its Applications
}

\author{
Changjian Zhi, Sanmin Wang, Yuantao Sun, and Bo Li \\ School of Mechanical Engineering, Northwestern Polytechnical University, Xian 710072, China \\ Correspondence should be addressed to Changjian Zhi; zhichangjiannwpu@163.com
}

Received 23 December 2014; Revised 11 March 2015; Accepted 12 March 2015

Academic Editor: Vladimir Turetsky

Copyright (c) 2015 Changjian Zhi et al. This is an open access article distributed under the Creative Commons Attribution License, which permits unrestricted use, distribution, and reproduction in any medium, provided the original work is properly cited.

\begin{abstract}
Constraint forces of the kinematic pair are the basis of the kinematics and dynamics analysis of mechanisms. Exploring the solution method for constraint forces is a hot issue in the mechanism theory fields. Based on the observation method and the theory of reciprocal screw system, the solution method of reciprocal screw system is improved and its solution procedures become easier. This method is also applied to the solution procedure of the constraint force. The specific expressions of the constraint force are represented by the reciprocal screw system of twist. The transformation formula of twist under different coordinates is given and it make the expression of the twist of kinematic pair more facility. A slider-crank mechanism and a single loop spatial RUSR mechanism are taken as examples. It confirms that this method can be used to solve the constraint force of the planar and spatial mechanism.
\end{abstract}

\section{Introduction}

The constraint force analysis of kinematic pair is not only the key of using mechanisms reasonably and creating new mechanisms but also the important factor of kinematics and dynamics analysis and the base of structure design of mechanism. Traditional methods of constraint force analysis of kinematic pair include the graphic method and analytical method [1]. Since they need drawing and the force figures, which go against programming, seeking new analysis methods is very necessary. Many scholars have a try in this filed and have got some achievements. Zhou et al. [2] utilizing the dismantle-bar method and the microdeformation and superposition principle analyzed the static force of the parallel mechanism. Zhao and Huang [3] using the analytical method analyzed the force of the lower-mobility parallel mechanism with overconstrained couple. Based on Zhao's research and screw theory, Liu et al. [4] analyzed the force of single loop spatial mechanism. Wang et al. [5] combining the traditional dismantle-bar method with screw theory analyzed the force of the spatial parallel mechanism. Jiang et al. [6] using Newton Euler method and D'Alembert principle established the force analysis equations, and they also put forward the dynamic analysis model of parallel mechanism based on the deformation compatibility method. Russo et al. [7] using the counterweight method and the springs method analyzed the static balancing of spatial parallel manipulator. Lu [8] used virtual work theory and CAD functionalities for solving active force and passive force of spatial parallel manipulators. Deepak and Ananthasuresh [9] used nonlinear springs to generate minimum torques which keep the cables taut and analyzed the static balancing of the parallel cable-driven mechanisms.

The reciprocal screw system represents the constraints and constraint forces acting on kinematic pairs. Since its solution method is complex and lacks commonality, based on Huang et al. [10] and Dai and Jones [11, 12] researches, we improve the solution method of the reciprocal screw system and make the solving process easier. In order to express constraint forces of the kinematic pair, the solution coefficient is introduced. Not only can this method solve constraint forces of the kinematic pair of mechanism but also it can analyze the static and dynamic force by combining it with the foregoing methods. We verify the method by solving constraint forces of the kinematic pair of a slider-crank mechanism and a single loop spatial RUSR mechanism. 


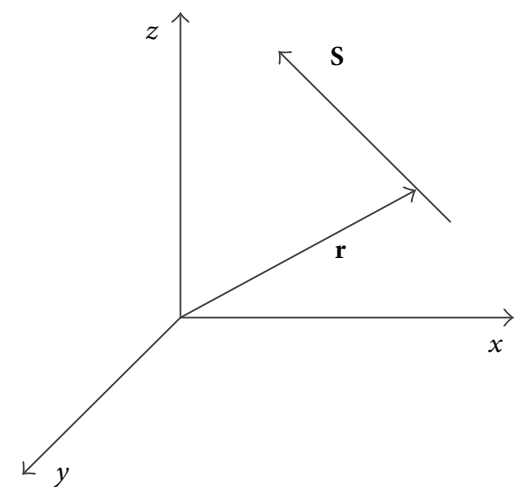

Figure 1: A line vector.

\section{Theoretic Foundation}

A line vector (Figure 1) can be denoted by a dual vector [10]

$$
\mathbf{S}^{\prime}=\left(\mathbf{S} ; \mathbf{S}_{0}\right)=(\mathbf{S} ; \mathbf{r} \times \mathbf{S})
$$

where $\left(\mathbf{S} ; \mathbf{S}_{0}\right)$ is called the Plücker coordinates, $\mathbf{S}$ is the real unit, which is the direction ratios of the line and is not origindependent, $\mathbf{S}_{0}$ is the real unit, which is the moment of the line about the origin and is origin-dependent, and $\mathbf{r}$ is the vector of a point on the line. $\mathbf{S}$ and $\mathbf{S}_{0}$ are three-dimensional vectors.

$\mathbf{S}$ and $\mathbf{S}_{0}$ satisfy the orthogonal condition, $\mathbf{S} \cdot \mathbf{S}_{0}=0$. When $\mathbf{S}=0$, the line passes through the origin. While $\mathbf{S}=0$, the line lies in a plane at infinity, and it becomes a couple.

If $\mathbf{S}$ and $\mathbf{S}_{0}$ do not satisfy the orthogonal condition, $\mathbf{S} \cdot \mathbf{S}_{0} \neq$ $0 . S^{\prime}$ is known as a screw. Ball [13] described that a screw is a straight line with the pitch. It can be expressed as

$$
\mathbf{S}^{\prime}=\left(\mathbf{S} ; \mathbf{S}^{0}\right)
$$

where $\mathbf{S}$ is the real unit, which is the direction ratios of the screw axis and is not origin-dependent, $\mathbf{S}^{0}$ is the dual unit, which is not origin-dependent. $\mathbf{S}$ and $\mathbf{S}^{0}$ are threedimensional vectors.

If $\mathbf{S} \neq 0$, the pitch of a screw is $h$ :

$$
h=\frac{\mathbf{S} \cdot \mathbf{S}^{0}}{\mathbf{S} \cdot \mathbf{S}} \text {. }
$$

In order to decide the position of the axis of a screw, $\mathbf{S}^{0}$ can be decomposed into two parts (Figure 2). One is parallel to $\mathbf{S}$ and the other is perpendicular to $\mathbf{S}$. Consider

$$
\mathbf{S}^{\prime}=\left(\mathbf{S} ; \mathbf{S}^{0}-h \mathbf{S}\right)+(\mathbf{0} ; h \mathbf{S}) \text {. }
$$

Since $\mathbf{S}^{0}-h \mathbf{S}$ is perpendicular to $\mathbf{S}, \mathbf{S}^{0}-h \mathbf{S}=\mathbf{S}_{0}$. $\left(\mathbf{S} ; \mathbf{S}^{0}-h \mathbf{S}\right)$ represents a line vector. $(\mathbf{S} ; h \mathbf{S})$ represents a couple. Obviously, a screw can be decomposed into a line vector and a couple. According to this formula, a line vector is a special screw.

The screw can be used to describe motions and forces. They are, respectively, called twist and wrench. The instantaneous twist of a rigid body can be written as

$$
\mathbf{S}^{\prime}=\left(\boldsymbol{\omega} ; \mathbf{v}^{0}\right)
$$

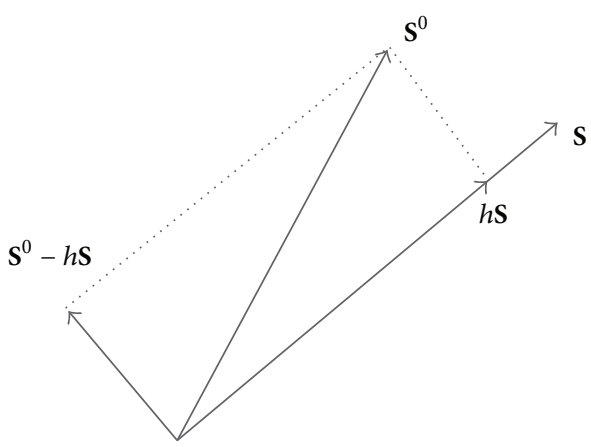

FIGURE 2: The axis of a screw.

where $\boldsymbol{\omega}$ is the instantaneous angular velocity of the rigid body and $\mathbf{v}^{0}$ is the instantaneous velocity a point in the rigid body coincident with the origin.

According to formula (4), $S^{\prime}$ can be written as

$$
\mathbf{S}^{\prime}=\left(\boldsymbol{\omega} ; \mathbf{v}^{0}-h \boldsymbol{\omega}\right)+(\mathbf{0} ; h \boldsymbol{\omega})
$$

where $\mathbf{v}^{0}-h \boldsymbol{\omega}=\mathbf{r} \times \boldsymbol{\omega} ; h$ can be obtained by formula (3).

Therefore, $\left(\boldsymbol{\omega} ; \mathbf{v}^{0}-h \boldsymbol{\omega}\right)$ can be seen as a pure rotation, and $(\mathbf{0} ; \boldsymbol{h} \boldsymbol{\omega})$ can be seen as a pure translation. A twist can be decomposed into a rotation and a translation.

Generally, all the spatial forces acting on a rigid body can be reduced to a force $\left(\mathbf{F} ; \mathbf{F}_{0}\right)$ and a couple $\left(\mathbf{0} ; \mathbf{T}^{0}\right)$. The force and the couple may have different directions. According to the screw algebra, the sum of the force and the couple is a new screw, which is called a wrench. Consider

$$
\mathbf{S}^{\prime}=\left(\mathbf{F} ; \mathbf{F}_{0}+\mathbf{T}^{0}\right)=\left(\mathbf{F} ; \mathbf{C}^{0}\right)
$$

According to formula (4), $\mathbf{S}^{\prime}$ can be written as

$$
\mathbf{S}^{\prime}=\left(\mathbf{F} ; \mathbf{C}^{0}-h \mathbf{F}\right)+(\mathbf{0} ; h \mathbf{F})
$$

where $\mathbf{C}^{0}-h \mathbf{F}=\mathbf{r} \times \mathbf{F} ; h$ can be obtained by formula (3).

Therefore, $\left(\mathbf{F} ; \mathbf{C}^{0}-h \mathbf{F}\right)$ can be seen as a force, and $(\mathbf{0} ; h \mathbf{F})$ can be seen as a couple. They have the same direction.

\section{Expressions of Constraint Forces of the Kinematic Pair of Mechanism by Screw}

The specific Plücker coordinate of twist and wrench can be written as [10]

$$
\mathbf{S}^{\prime}=\left(L, M, N ; P^{*}, Q^{*}, R^{*}\right),
$$

where $\mathbf{S}=(L, M, N), \mathbf{S}^{0}=\left(L^{*}, M^{*}, N^{*}\right)$.

If $\mathbf{S}^{\prime}$ represents a twist, $(L, M, N)$ is its angular velocity, and $\left(L^{*}, M^{*}, N^{*}\right)$ is its linear velocity.

If $\mathbf{S}^{\prime}$ represents a wrench, $(L, M, N)$ is its force part, and $\left(L^{*}, M^{*}, N^{*}\right)$ is its couple part.

In order to calculate the reciprocal screw, a screw can be expressed by a row vector:

$$
\mathbf{S}^{\prime}=\left[\begin{array}{llllll}
L & M & N & P^{*} & Q^{*} & R^{*}
\end{array}\right] .
$$


A kinematic pair is a combination of two kinematic bodies which have relative motion with respect to each other. Its motion can be described by screw system. The order of screw system is the same as the mobility. If the mobility of a kinematic pair is $n$ ( $1 \leq n \leq 5, n$ is an integer), its twist is expressed as

$$
\mathbf{S}^{\prime}=\left[\begin{array}{cccccc}
L_{1} & M_{1} & N_{1} & P_{1}^{*} & Q_{1}^{*} & R_{1}^{*} \\
\vdots & & & & & \vdots \\
L_{n} & M_{n} & N_{n} & P_{n}^{*} & Q_{n}^{*} & R_{n}^{*}
\end{array}\right] .
$$

$\mathbf{S}^{\prime}$ is a screw system, and its reciprocal screw system is $\mathbf{S}^{\mathbf{r}}$. They satisfy the following relationship:

$$
\mathbf{S}^{\prime} \circ \mathbf{S}^{\prime \mathbf{r}}=0,
$$

where $\circ$ is the reciprocal product.

Let $\mathbf{V}=\mathbf{S}^{\prime \mathbf{r}} \Delta$, where $\Delta$ is transformation operator between the real unit vector and the dual unit vector. The expression of $\Delta$ is

$$
\Delta=\left[\begin{array}{ll}
\mathbf{0} & \mathbf{I} \\
\mathbf{I} & \mathbf{0}
\end{array}\right],
$$

where $\mathbf{I}^{\prime}$ is a 3 by 3 unit matrix.

$\mathbf{S}^{\prime}$ and $\mathbf{V}$ satisfy the following relationship:

$$
\mathbf{S}^{\prime} \mathbf{V}=0
$$

V can be regarded as the null space of the screw systems $\mathbf{S}^{\prime}$. Based on Huang's observation method and Dai's linear algebraic method obtaining reciprocal screw systems, we improve the solution method of $\mathbf{V}$. The explicit procedures are as follows.

(1) The solution procedures for the row vectors of $\mathbf{V}$ which correspond to the zero column vectors of screw systems $\mathbf{S}^{\prime}$ can be described as follows.

There are $e(0 \leq e \leq 5)$ zero column vectors in the screw systems $\mathbf{S}^{\prime}$. D is a submatrix which is the preceding $e$ rows of $\mathbf{V} . \mathbf{D}_{r s}$ is the element of $\mathbf{D}$ in the $r$ th row and $s$ th column, $1 \leq r \leq e, 1 \leq s \leq 6$. $s$ is the numerical order of the $r$ th zero column vector in the $\mathbf{S}^{\prime}$. In this method, $\mathbf{D}_{r s}$ equals 1 , and the rest of elements of $\mathbf{D}$ equal 0 .

(2) The solution procedures of the rest of row vectors of $\mathrm{V}$ are as follows.

(i) The solution of the $i$ th row vector of $\mathbf{V}, e+1 \leq i \leq$ $6-n \cdot n+1$ columns where the zero column vector is excluded are chosen from $\mathbf{S}^{\prime}$ forming the matrix $\mathbf{C}$. If $i$ is different, the chosen column vectors are different. The numerical orders of the chosen columns are saved in the $i$ th row of the matrix $\mathbf{O}$ in order which is a $(6-n)$ by $(n+1)$ matrix.

(ii) If there are no zero rows in the matrix $\mathbf{C}$, the following equation is established:

$$
\mathbf{G}_{i j}=(-1)^{n+i+j}\left|\mathbf{C}_{j}\right| \quad(1 \leq j \leq n+1),
$$

where $\mathbf{G}$ is a $(6-n)$ by $(n+1)$ matrix; $\mathbf{C}_{j}$ is a new matrix which is formed by deleting the $j$ th column of the $\mathbf{C}$ matrix. The element of $\mathbf{V}$ in the $i$ th row and $k$ th column is $\mathbf{V}_{i k}$. It satisfies the following relationship:

$$
\mathbf{V}_{i k}=\mathbf{G}_{i j}
$$

where $k=\mathbf{O}_{i j}$.

The rest of elements of the $i$ th column of $\mathbf{V}$ equal 0 .

If there is a zero row vector in the matrix $\mathbf{C}$, it is removed forming a new matrix $\mathbf{M}$. $n$ columns are chose from $\mathbf{M}$ which forms a $(n-1)$ by $n$ matrix $\mathbf{N}$. The numerical order of the chosen columns in the matrix $\mathbf{S}^{\prime}$ are saved in the $i$ th row of the matrix $\mathbf{H}$ which is a $(6-n)$ by $n$ matrix. Consider

$$
\mathbf{G}_{i p}=(-1)^{n+i+p}\left|\mathbf{N}_{p}\right| \quad(1 \leq p \leq n),
$$

where $\mathbf{G}$ is a $(6-n)$ by $(n+1)$ matrix; $\mathbf{N}_{p}$ is a new matrix which is formed by deleting the $p$ th column of the $\mathbf{N}$ matrix. The element of $\mathbf{V}$ in the $i$ th row and $k$ th column is $\mathbf{V}_{i k}$. It satisfies the following relationship:

$$
\mathbf{V}_{i k}=\mathbf{G}_{i p}
$$

where $k=\mathbf{H}_{i j}$.

The rest of elements of the $i$ th column of $\mathbf{V}$ equal 0 .

(iii) If $\mathbf{V}$ can satisfy the requirements, the solution procedures of the reciprocal screw system of $\mathbf{S}^{\prime}$ are finished. If $\mathbf{V}$ cannot satisfy the requirements, the dissatisfactory row is removed and step (2) is repeated until $\mathbf{V}$ can satisfy the requirements.

The reciprocal screw system of twist system is expressed as

$$
\mathbf{S}^{\prime r}=\mathbf{V} \Delta
$$

The constraint wrench of the kinematic pair is written as

$$
\mathbf{S}_{F}=f_{1} \mathbf{S}_{1}^{\prime r}+f_{2} \mathbf{S}_{2}^{\prime r}+\cdots+f_{6-n} \mathbf{S}_{6-n}^{\prime r},
$$

where $f_{i}(i=1, \ldots, 6-n)$ is the solution coefficient of the constraint wrench.

\section{The Transformation of Twist of Kinematic Pair between Different Coordinate Frames}

Expressions of twist of kinematic pair of spatial mechanism are hard to be obtained. It is necessary to set up a new coordinate frame. The rotation and displacement transformation matrix from the coordinate frames 2 to 1 are, respectively, $\mathbf{R}$ and $\mathbf{d}$.

The twist of a kinematic pair in the coordinate frame 2 is written as

$$
\mathbf{S}_{2}^{\prime}=\left(\mathbf{S}_{2} ; \mathbf{S}_{2}^{0}\right) \text {. }
$$




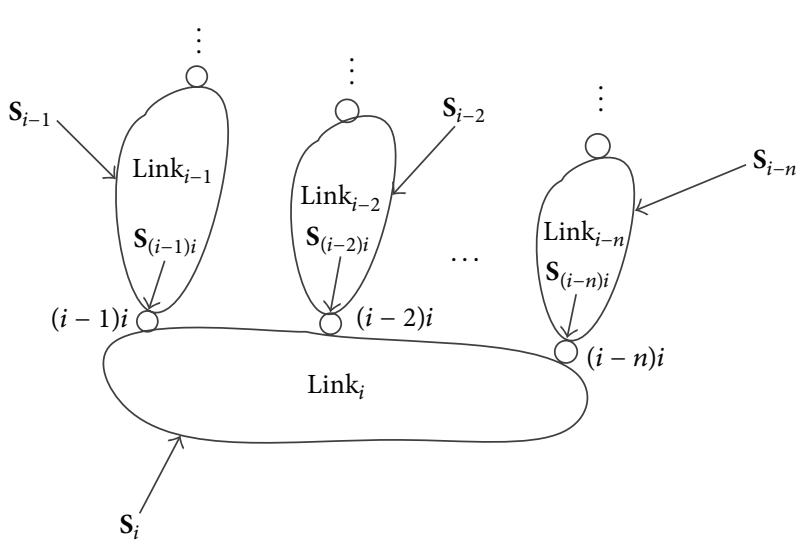

FIgURE 3: The force figure of link $i$.

It can be divided into two parts:

$$
\begin{gathered}
\mathbf{S}_{21}^{\prime}=\left(\mathbf{S}_{2} ; \mathbf{r}_{2} \times \mathbf{S}_{2}\right), \\
\mathbf{S}_{22}^{\prime}=\left(\mathbf{0} ; h \mathbf{S}_{2}\right) .
\end{gathered}
$$

The twist in the coordinate frame 1 is expressed as

$$
\mathbf{S}_{1}^{\prime}=\left(\mathbf{S}_{1} ; \mathbf{S}_{1}^{0}\right)
$$

It can be divided into two parts:

$$
\begin{gathered}
\mathbf{S}_{11}^{\prime}=\left(\mathbf{S}_{1} ; \mathbf{r}_{1} \times \mathbf{S}_{1}\right), \\
\mathbf{S}_{12}^{\prime}=\left(0 ; h \mathbf{S}_{1}\right),
\end{gathered}
$$

where $\mathbf{S}_{1}=\mathbf{R} \mathbf{S}_{2}, \mathbf{r}_{1}=\mathbf{R} \mathbf{r}_{2}+\mathbf{d}$.

The twist in the coordinate frame 1 also can be expressed as

$$
\mathbf{S}_{1}^{\prime}=\left(\mathbf{R S}_{2} ;\left(\mathbf{R r}_{2}+\mathbf{d}\right) \times\left(\mathbf{R S}_{2}\right)+h\left(\mathbf{R S}_{2}\right)\right)
$$

\section{Equilibrium Equations of Links}

In the mechanism, wrenches acting on links can be classified into two types. One is the constraint wrenches of kinematic pair and the other is the external wrench. In Figure 3, link $i$ which is a part of a mechanism has $n$ kinematic pairs. When it is in an equilibrium state, the following equation is established $[14,15]$ :

$$
\mathbf{S}_{(i-1) i}+\mathbf{S}_{(i-2) i}+\cdots+\mathbf{S}_{(i-n) i}+\mathbf{S}_{i}=0,
$$

where $\mathbf{S}_{(i-k) i}$ is the constraint wrench acted by link $(i-k) ; \mathbf{S}_{i}$ is the resultant external wrench.

\section{Numerical Examples}

6.1. The Solution for Constraint Wrenches of Kinematic Pairs in the Planar Mechanism. The common kinematic pairs in the planar mechanism mainly have the revolute pair and the prismatic pair. In the coordinate frame $o-x y z$, the twist of the revolute pair and the prismatic pair are, respectively, $\mathbf{S}_{R}^{\prime}$ and $\mathbf{S}_{M}^{\prime}$ :

$$
\begin{gathered}
\mathbf{S}_{R}^{\prime}=\left[\begin{array}{llllll}
0 & 0 & 1 & y & -x & 0
\end{array}\right] \\
\mathbf{S}_{M}^{\prime}=\left[\begin{array}{llllll}
0 & 0 & 0 & \cos (\tau) & \sin (\tau) & 0
\end{array}\right]
\end{gathered}
$$

where $\tau$ is the included angle between the displacement direction of the prismatic pair and the $x$-axis direction.

The reciprocal screw system of the rotation pair of planar mechanism is given by

$$
\mathbf{S}_{R}^{\prime r}=\left[\begin{array}{cccccc}
1 & 0 & 0 & 0 & 0 & -y \\
0 & 1 & 0 & 0 & 0 & x \\
0 & 0 & 1 & 0 & 0 & 0 \\
0 & 0 & 0 & 1 & 0 & 0 \\
0 & 0 & 0 & 0 & 1 & 0
\end{array}\right]
$$

According to formulas (20) and (28), the constraint wrench of the rotation pair in the planar mechanism is given by

$$
\mathbf{S}_{F}=\left[\begin{array}{llllll}
f_{1} & f_{2} & f_{3} & f_{4} & f_{5} & -f_{1} y+f_{2} x
\end{array}\right] .
$$

The component force of the $z$ direction of the rotation pair in the plane xoy and the moment about $x$-and $y$-axis equals zero. Therefore, $f_{3}=f_{4}=f_{5}=0$. The reciprocal screw system of the rotation pair can be simplified as

$$
\mathbf{S}^{\prime r}=\left[\begin{array}{cccccc}
1 & 0 & 0 & 0 & 0 & -y \\
0 & 1 & 0 & 0 & 0 & x
\end{array}\right]
$$

According to formulas (20) and (28), the constraint wrench of the rotation pair in the planar mechanism is expressed as

$$
\mathbf{S}_{F}^{\prime}=f_{1} \mathbf{S}_{1}^{\prime r}+f_{2} \mathbf{S}_{2}^{\prime r}
$$

Similarly, the reciprocal screw system of the prismatic pair can be simplified as

$$
\mathbf{S}^{\prime r}=\left[\begin{array}{cccccc}
-\sin (\tau) & \cos (\tau) & 0 & 0 & 0 & 0 \\
0 & 0 & 0 & 0 & 0 & 1
\end{array}\right],
$$

where $\tau$ is the included angle between the displacement direction of the prismatic pair and the $x$-axis direction.

According to formulas (20) and (28), the constraint wrench of the planar prismatic pair is shown as

$$
\mathbf{S}_{F}^{\prime}=f_{1} \mathbf{S}_{1}^{\prime r}+f_{2} \mathbf{S}_{2}^{\prime r} \text {. }
$$

In order to verify the validity of the foregoing method, a slider-crank mechanism (Figure 4) is taken as an example.

The known geometry conditions are as follows: $\tau=$ $0^{\circ}, O A=100 \mathrm{~mm}$, and $A B=400 \mathrm{~mm}$. In this paper, the units of force and moment are, respectively, $\mathrm{N}$ and $\mathrm{N} \cdot \mathrm{mm}$. 


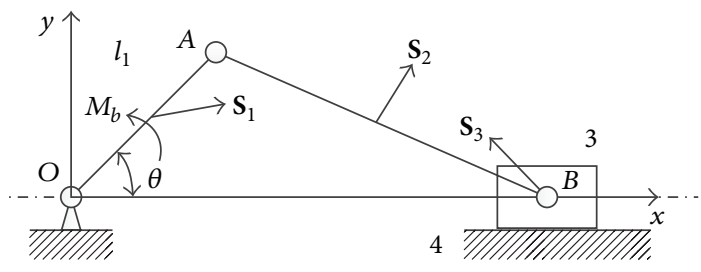

FIgURE 4: The slider-crank mechanism.

The resultant external wrenches acting on each link and the trimming moment acting on link 1 are, respectively, given by

$$
\begin{aligned}
\mathbf{S}_{1} & =\left[\begin{array}{llllll}
30 & 40 & 0 & 0 & 0 & -270
\end{array}\right] \\
\mathbf{S}_{2} & =\left[\begin{array}{llllll}
25 & 54 & 0 & 0 & 0 & 440
\end{array}\right] \\
\mathbf{S}_{3} & =\left[\begin{array}{llllll}
-24 & 67 & 0 & 0 & 0 & 0
\end{array}\right] \\
\mathbf{S}_{b} & =\left[\begin{array}{llllll}
0 & 0 & 0 & 0 & 0 & M_{d}
\end{array}\right]
\end{aligned}
$$

Twists of kinematic pairs in the mechanism are written as

$$
\begin{aligned}
& \mathbf{s}_{O}^{\prime}=\left[\begin{array}{llllll}
0 & 0 & 1 & y_{O} & -x_{O} & 0
\end{array}\right], \\
& \mathbf{s}_{A}^{\prime}=\left[\begin{array}{llllll}
0 & 0 & 1 & y_{A}-x_{A} & 0
\end{array}\right], \\
& \mathbf{s}_{B}^{\prime}=\left[\begin{array}{llllll}
0 & 0 & 1 & y_{B} & -x_{B} & 0
\end{array}\right], \\
& \mathbf{s}_{C}^{\prime}=\left[\begin{array}{llllll}
0 & 0 & 0 & y_{C} & -x_{C} & 0
\end{array}\right] .
\end{aligned}
$$

The reciprocal screw systems of kinematic pairs in the mechanism are given by

$$
\begin{aligned}
\mathbf{S}_{O}^{\prime r} & =\left[\begin{array}{cccccc}
1 & 0 & 0 & 0 & 0 & -y_{O} \\
0 & 1 & 0 & 0 & 0 & x_{O}
\end{array}\right], \\
\mathbf{S}_{A}^{\prime r} & =\left[\begin{array}{lllllc}
1 & 0 & 0 & 0 & 0 & -y_{A} \\
0 & 1 & 0 & 0 & 0 & x_{A}
\end{array}\right], \\
\mathbf{S}_{B}^{\prime r} & =\left[\begin{array}{llllll}
1 & 0 & 0 & 0 & 0 & -y_{B} \\
0 & 1 & 0 & 0 & 0 & x_{B}
\end{array}\right], \\
\mathbf{S}_{C}^{\prime r} & =\left[\begin{array}{llllll}
0 & 1 & 0 & 0 & 0 & 0 \\
0 & 0 & 0 & 0 & 0 & 1
\end{array}\right] .
\end{aligned}
$$

According to formulas (29), (31), and (33), the constraint wrenches of kinematic pairs are as follows:

$$
\begin{gathered}
\mathbf{S}_{F O}=\left[\begin{array}{lllllll}
f_{1} & f_{2} & 0 & 0 & 0 & 0
\end{array}\right], \\
\mathbf{S}_{F A}=\left[\begin{array}{lllllll}
f_{3} & f_{4} & 0 & 0 & 0 & 86.6 f_{4}-50 f_{3}
\end{array}\right], \\
\mathbf{S}_{F B}=\left[\begin{array}{llllll}
f_{5} & f_{6} & 0 & 0 & 0 & 480.3 f_{6}
\end{array}\right], \\
\mathbf{S}_{F C}=\left[\begin{array}{llllll}
0 & f_{7} & 0 & 0 & 0 & f_{8}
\end{array}\right] .
\end{gathered}
$$

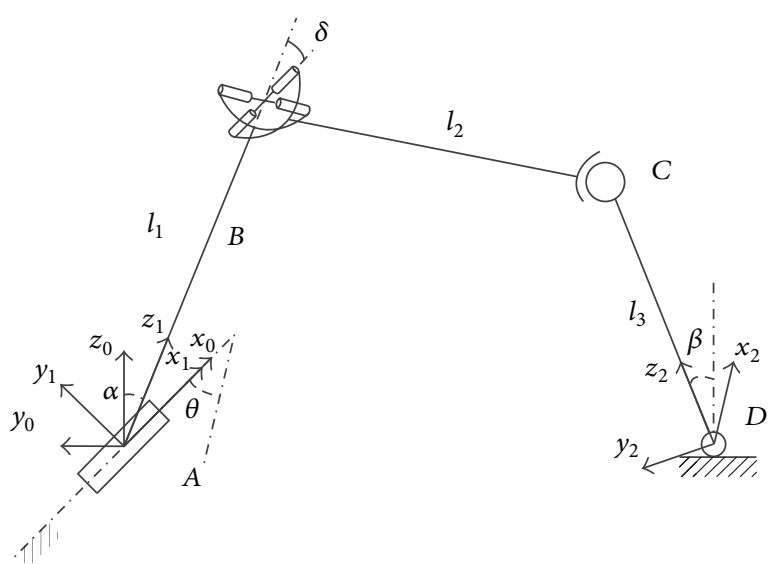

FIGURE 5: The single loop spatial RUSR mechanism.

Based on the formula (26) and Figure 3, the analysis model of the constraint wrenches of kinematic pairs obtained

$$
\begin{gathered}
\mathbf{S}_{F O}+\mathbf{S}_{1}+\mathbf{S}_{b}-\mathbf{S}_{F A}=0 \\
\mathbf{S}_{F A}+\mathbf{S}_{2}-\mathbf{S}_{F B}=0 \\
\mathbf{S}_{F B}+\mathbf{S}_{3}-\mathbf{S}_{F C}=0 .
\end{gathered}
$$

Each wrench equilibrium equation can expand to 3 equations. Therefore, 9 equations can be obtained in total. Since there are 8 unknown solution coefficients and a trimming moment, the number of unknowns equals the number of equations. The analysis model can be solved. The constraint wrenches of kinematic pairs and the trimming moment of the slider-crank mechanism can be obtained:

$$
\begin{gathered}
\mathbf{S}_{F O}=\left[\begin{array}{lllllll}
-31.0 & -104.6 & 0 & 0 & 0 & 0
\end{array}\right], \\
\mathbf{S}_{F A}=\left[\begin{array}{llllll}
-1.0 & -64.6 & 0 & 0 & 0 & -5547.5
\end{array}\right], \\
\mathbf{S}_{F B}=\left[\begin{array}{llllll}
24 & -10.6 & 0 & 0 & 0 & -5107.5
\end{array}\right], \\
\mathbf{S}_{F C}=\left[\begin{array}{llllll}
0 & 56.4 & 0 & 0 & 0 & -5107.5
\end{array}\right] \\
\mathbf{S}_{b}=\left[\begin{array}{llllll}
0 & 0 & 0 & 0 & 0 & -5277.5
\end{array}\right]
\end{gathered}
$$

6.2. The Solution for the Constraint Wrenches of the Universal Spatial Kinematic Pairs. The universal spatial kinematic pairs include the revolute pair, universal joint, spherical pair, and prismatic pair. In order to learn the solution method of the constraint wrenches in the spatial kinematic pairs, the single loop spatial RUSR mechanism is taken as example. Its schematic figure is shown in Figure 5. $A$ and $D$ are the revolute pairs. $B$ and $C$ are, respectively, the universal joint and the spherical pair. In order to express twists of kinematic pairs easily, the coordinate frame $A-x_{0} y_{0} z_{0}$, the local coordinate frame $A-x_{1} y_{1} z_{1}$, and the local coordinate frame $D-x_{2} y_{2} z_{2}$ are set up. The axes $x_{0}$ and $x_{1}$ are coincident with the axis direction of $A$, and the axis $z_{1}$ is coincident with link 1 . The included angle between $z_{1}$ and $z_{0}$ is $\alpha$. The included angle between $x_{2}$ and $x_{0}$ is $\theta$. The included angle between $z_{2}$ and $z_{0}$ is $\beta$. 
The known conditions are as follows: $l_{1}=250 \mathrm{~mm}, l_{2}=$ $350 \mathrm{~mm}, l_{3}=150 \mathrm{~mm}$, and $l_{4}=500 \mathrm{~mm}$ and $\theta=30^{\circ}, \alpha=60^{\circ}$, $\beta=60^{\circ}$, and $\delta=30^{\circ}$. The resultant external wrenches acting on link 2 and link 3 are, respectively,

$$
\begin{aligned}
& \mathbf{S}_{2}=\left[\begin{array}{llllll}
-60 & 52 & -42 & 230 & 320 & 440
\end{array}\right] \\
& \mathbf{S}_{3}=\left[\begin{array}{llllll}
-54 & 67 & 21 & 268 & -270 & 132
\end{array}\right]
\end{aligned}
$$

The coordinates of each kinematic pairs in the different coordinate frames are given by

$$
\begin{aligned}
A_{0} & =\left(\begin{array}{lll}
0 & 0 & 0
\end{array}\right), \\
B_{1} & =\left(\begin{array}{lll}
0 & 0 & l_{1}
\end{array}\right), \\
C_{2} & =\left(\begin{array}{lll}
0 & 0 & l_{3}
\end{array}\right), \\
D_{0} & =\left(\begin{array}{lll}
0 & -l_{4} & 0
\end{array}\right),
\end{aligned}
$$

where the subscripts represent the numerical order of coordinate frames.

In the following parts, notations are defined as

$$
\begin{gathered}
Z_{1}=\sin (\delta), \\
Z_{2}=\cos (\delta), \\
Z_{3}=\sin (\beta), \\
Z_{4}=\cos (\beta), \\
Z_{5}=\sin (\theta), \\
Z_{6}=\cos (\theta), \\
Z_{7}=\sin (\alpha), \\
Z_{8}=\cos (\alpha), \\
Z_{9}=\sin (\alpha+\delta), \\
Z_{10}=\cos (\alpha+\delta), \\
Z_{11}=l_{3} \sin (\beta) \cos (\theta)+l_{4} .
\end{gathered}
$$

Twists of kinematic pairs in the different coordinate frames are shown as

$$
\begin{gathered}
\mathbf{s}_{A 0}^{\prime}=\left[\begin{array}{llllll}
1 & 0 & 0 & 0 & 0 & 0
\end{array}\right], \\
\mathbf{S}_{B 1}^{\prime}=\left[\begin{array}{cccccc}
1 & 0 & 0 & 0 & l_{1} & 0 \\
0 & -Z_{1} & Z_{2} & l_{1} Z_{1} & 0 & 0
\end{array}\right], \\
\mathbf{s}_{C 0}^{\prime}=\left[\begin{array}{cccccc}
1 & 0 & 0 & 0 & l_{3} Z_{4} & l_{3} Z_{3} Z_{6}+l_{4} \\
0 & 1 & 0 & -l_{3} Z_{4} & 0 & l_{3} Z_{3} Z_{5} \\
0 & 0 & 1 & -l_{3} Z_{3} Z_{6}-l_{4} & -l_{3} Z_{3} Z_{5} & 0
\end{array}\right], \\
\mathbf{S}_{D 2}^{\prime}=\left[\begin{array}{llllll}
1 & 0 & 0 & 0 & 0 & 0
\end{array}\right] .
\end{gathered}
$$

The rotation transformation matrix from coordinate frames 2 to 1 are, respectively, shown as follows:

$$
\mathbf{T}_{10}=\left[\begin{array}{ccc}
1 & 0 & 0 \\
0 & Z_{8} & -Z_{7} \\
0 & Z_{7} & Z_{8}
\end{array}\right], \quad \mathbf{T}_{20}=\left[\begin{array}{ccc}
Z_{6} & -Z_{4} Z_{5} & Z_{3} Z_{5} \\
Z_{5} & Z_{4} Z_{6} & -Z_{3} Z_{6} \\
0 & Z_{3} & Z_{4}
\end{array}\right] \text {. }
$$

The displacement transformation matrix from coordinate frames 2 to 1 are, respectively, shown as

$$
\mathbf{P}_{10}=\left[\begin{array}{l}
0 \\
0 \\
0
\end{array}\right], \quad \mathbf{P}_{20}=\left[\begin{array}{c}
0 \\
-l_{4} \\
0
\end{array}\right]
$$

According to formula (25), the coordinates and the twist system of each kinematic pair are obtained:

$$
\begin{gathered}
B_{0}=\left(\begin{array}{cccc}
-l_{1} Z_{7} & l_{1} Z_{8} & 0
\end{array}\right), \\
C_{0}=\left(\begin{array}{llllll}
l_{3} Z_{3} Z_{5} & -l_{3} Z_{3} Z_{6} & -l_{4} & l_{3} Z_{4}
\end{array}\right), \\
\mathbf{S}_{B 0}^{\prime}=\left[\begin{array}{cccccc}
1 & 0 & 0 & 0 & l_{1} Z_{8} & l_{1} Z_{7} \\
0 & -Z_{9} & Z_{10} & l_{1} Z_{1} & 0 & 0
\end{array}\right], \\
\mathbf{S}_{D 0}^{\prime}=\left[\begin{array}{llllll}
Z_{6} & Z_{5} & 0 & 0 & 0 & l_{4} Z_{6}
\end{array}\right] .
\end{gathered}
$$

Based on the improved solution method of $\mathbf{V}$, the reciprocal screw systems of kinematic pairs are shown as

$$
\begin{aligned}
& \mathbf{S}_{A 0}^{\prime r}=\left[\begin{array}{llllll}
1 & 0 & 0 & 0 & 0 & 0 \\
0 & 1 & 0 & 0 & 0 & 0 \\
0 & 0 & 1 & 0 & 0 & 0 \\
0 & 0 & 0 & 0 & 1 & 0 \\
0 & 0 & 0 & 0 & 0 & 1
\end{array}\right], \\
& \mathbf{S}_{B 0}^{\prime r}=\left[\begin{array}{cccccc}
0 & 0 & 0 & 0 & -Z_{10} & -Z_{9} \\
0 & l_{1} Z_{1} & 0 & -l_{1}^{2} Z_{1} Z_{8} & 0 & 0 \\
l_{1} Z_{10} Z_{8} & 0 & 0 & 0 & 0 & -l_{1}^{2} Z_{1} Z_{8} \\
0 & -l_{1}^{2} Z_{1} Z_{7} & l_{1}^{2} Z_{1} Z_{8} & 0 & 0 & 0
\end{array}\right] \text {, }
\end{aligned}
$$




$$
\begin{gathered}
\mathbf{s}_{\mathrm{C} 0}^{\prime r}=\left[\begin{array}{cccccc}
1 & 0 & 0 & 0 & l_{3} Z_{4} & Z_{11} \\
-l_{3} Z_{3} Z_{5} & Z_{11} & 0 & l_{3} Z_{4} Z_{11} & -l_{3}^{2} Z_{4} Z_{3} Z_{5} & 0 \\
l_{3}^{2} Z_{4} Z_{3} Z_{5} & -l_{3} Z_{4} Z_{11} & l_{3}^{2} Z_{4}^{2} & 0 & 0 & 0
\end{array}\right], \\
\mathbf{s}_{D 0}^{\prime r}=\left[\begin{array}{cccccc}
1 & 0 & 0 & 0 & 0 & 0 \\
0 & 1 & 0 & 0 & 0 & 0 \\
0 & 0 & 0 & 0 & 0 & 1 \\
0 & 0 & 0 & Z_{5} & -Z_{6} & 0 \\
0 & 0 & -Z_{5} & 0 & l_{4} Z_{6} & 0
\end{array}\right]
\end{gathered}
$$

According to formula (20), the constraint wrenches of all kinematic pairs in the spatial mechanism are obtained:

$$
\begin{gathered}
\mathbf{s}_{A F}=\left[\begin{array}{llllll}
f_{1} & f_{2} & f_{3} & 0 & f_{4} & f_{5}
\end{array}\right], \\
\mathbf{S}_{B F}=\left[\begin{array}{lllllll}
f_{8} l_{1} Z_{10} Z_{8} & f_{7} l_{1} Z_{1}-f_{9} l_{1}^{2} Z_{1} Z_{7} & f_{9} l_{1}^{2} Z_{1} Z_{8} & -f_{6} l_{1}^{2} Z_{1} Z_{8} & -f_{5} Z_{10} & -f_{5} Z_{9}-f_{7} l_{1}^{2} Z_{1} Z
\end{array}\right], \\
\mathbf{s}_{C F}=\left[\begin{array}{ccccccc}
f_{10}-f_{11} l_{3} Z_{3} Z_{5}+f_{12} l_{3}^{2} Z_{3} Z_{4} Z_{5} & f_{11} Z_{11}-f_{12} l_{3} Z_{11} Z_{4} & f_{12} l_{3}^{2} Z_{4}^{2} & f_{11} l_{3} Z_{4} Z_{11} & f_{10} l_{3} Z_{4}-f_{11} l_{3}^{2} Z_{4} Z_{3} Z_{5} & f_{10} Z_{11}
\end{array}\right], \\
\mathbf{S}_{D F}=\left[\begin{array}{llllll}
f_{13} & f_{14} & -f_{17} Z_{5} & f_{16} Z_{5} & -f_{16} Z_{6}+f_{17} l_{4} Z_{6} & f_{15}
\end{array}\right] .
\end{gathered}
$$

According to formula (26) and Figure 3, the analysis model of the constraint wrenches of the single loop RUSR mechanism is obtained:

$$
\begin{gathered}
\mathbf{S}_{A F}+\mathbf{S}_{1}+\mathbf{S}_{b}-\mathbf{S}_{B F}=0, \\
\mathbf{S}_{B F}+\mathbf{S}_{2}-\mathbf{S}_{C F}=0, \\
\mathbf{S}_{C F}+\mathbf{S}_{3}-\mathbf{S}_{D F}=0 .
\end{gathered}
$$

Equations (49) can expand into 18 equations. Since there are 17 unknown solution coefficients and a trimming moment, the number of unknowns equals the number of equations. The analysis model is solvable. The constraint wrenches of kinematic pairs and the e trimming moment of the slider-crank mechanism are shown as follows:

$$
\begin{gathered}
\mathbf{S}_{A F}=\left[\begin{array}{llllll}
2251.5 & 5040.0 & -682.3 & 0 & -8.2 & 989.1
\end{array}\right], \\
\mathbf{S}_{B F}=\left[\begin{array}{llllll}
2251.5 & 5040.0 & -682.3 & -482.3 & -8.2 & 989.1
\end{array}\right], \\
\mathbf{S}_{C F}=\left[\begin{array}{llllll}
2191.5 & 5092.0 & -724.3 & -252.3 & 311.8 & 1429.1
\end{array}\right], \\
\mathbf{S}_{D F}=\left[\begin{array}{llllll}
2137.5 & 5159.0 & -703.3 & 15.7 & 581.8 & 1561.1
\end{array}\right] .
\end{gathered}
$$

\section{Conclusion}

(1) The constraint wrenches of kinematic pairs can be expressed by the reciprocal screw system. Based on this fact, the solution method of constraint wrenches is formed.
(2) The improved solution can solve the reciprocal screw system by the programming conveniently and swiftly. It can enhance the solution efficiency of constraint wrenches.

(3) The constraint wrench has an important significance for the analysis and application of the mechanism. According to the examples mentioned in this paper, the solution method of the constraint wrenches of kinematic pairs, which is based on the reciprocal screw, can solve the constraint wrenches of the planar and spatial mechanism.

\section{Conflict of Interests}

The authors declare that there is no conflict of interests regarding the publication of this paper.

\section{Acknowledgment}

The authors gratefully acknowledge the financial support of the National Natural Science Foundation of China (Grant no. 51175422).

\section{References}

[1] J. J. Uicker, G. R. Pennock, and J. E. Shigley, Theory of Machines and Mechanisms, Oxford University Press, Oxford, UK, 2011.

[2] Y. Zhou, L. Liu, and F. Gao, "Static full-solutions of spherical parallel mechanism 3-RRR with 3-DOF," Chinese Journal of Mechanical Engineering, vol. 44, no. 6, pp. 169-176, 2008. 
[3] Y. Zhao and Z. Huang, "Force analysis of lower-mobility parallel mechanisms with over-constrained couples," Journal of Mechanical Engineering, vol. 46, no. 5, pp. 15-21, 2010.

[4] S. C. Liu, Z. M. Chen, J. F. Liu, and Z. Huang, "Force analysis of single ring spatial RSUR mechanism based on screw theory," Journal of Mechanical Engineering, vol. 47, no. 13, pp. 23-28, 2011.

[5] G. X. Wang, H. Z. Liu, D. N. Yuan et al., "Force analysis of spatial 4-SPS /CU parallel mechanism," Transactions of the Chinese Society of Agricultural Engineering, vol. 28, no. 22, pp. 30-38, 2012.

[6] Y. Jiang, T. M. Li, and L. P. Wang, "Research on the dynamic model of an over-constrained parallel mechanism," Journal of Mechanical Engineering, vol. 49, no. 17, pp. 123-129, 2013.

[7] A. Russo, R. Sinatra, and F. Xi, "Static balancing of parallel robots," Mechanism and Machine Theory, vol. 40, no. 2, pp. 191202, 2005.

[8] Y. Lu, "Using virtual work theory and CAD functionalities for solving active force and passive force of spatial parallel manipulators," Mechanism and Machine Theory, vol. 42, no. 7, pp. 839-858, 2007.

[9] S. R. Deepak and G. K. Ananthasuresh, "Static balancing of a four-bar linkage and its cognates," Mechanism and Machine Theory, vol. 48, no. 1, pp. 62-80, 2012.

[10] Z. Huang, Q. Li, and H. Ding, Theory of Parallel Mechanisms, Springer, Berlin, Germany, 2012.

[11] J. S. Dai and J. R. Jones, "A linear algebraic procedure in obtaining reciprocal screw systems," Journal of Robotic Systems, vol. 20, no. 7, pp. 401-412, 2003.

[12] J. S. Dai and J. R. Jones, "Null-space construction using cofactors from a screw-algebra context," Proceedings of the Royal Society of London, Series A: Mathematical, Physical and Engineering Sciences, vol. 458, no. 2024, pp. 1845-1866, 2002.

[13] R. S. Ball, A Treatise on the Theory of Screws, Cambridge University Press, Cambridge, UK, 1998.

[14] M. J. Tsai and T. S. Lai, "Door closing force analysis of WattI type hinge with joint clearance," in Proceedings of the 12th IFToMM World Congress, pp. 18-21, Besançon, France, June 2007.

[15] M.-J. Tsai and T.-H. Lai, "Accuracy analysis of a multi-loop linkage with joint clearances," Mechanism and Machine Theory, vol. 43, no. 9, pp. 1141-1157, 2008. 


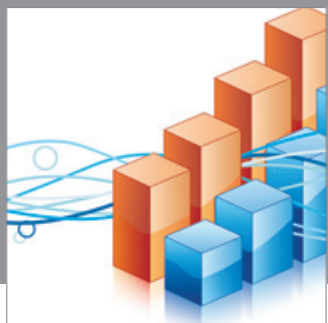

Advances in

Operations Research

mansans

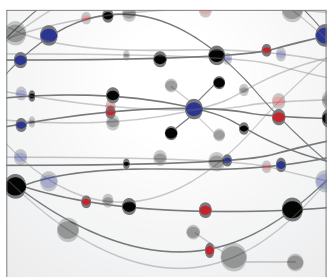

The Scientific World Journal
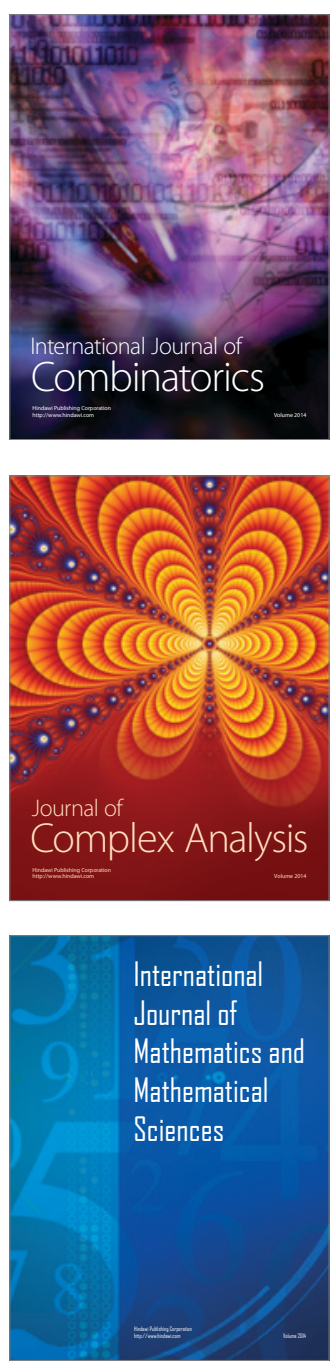
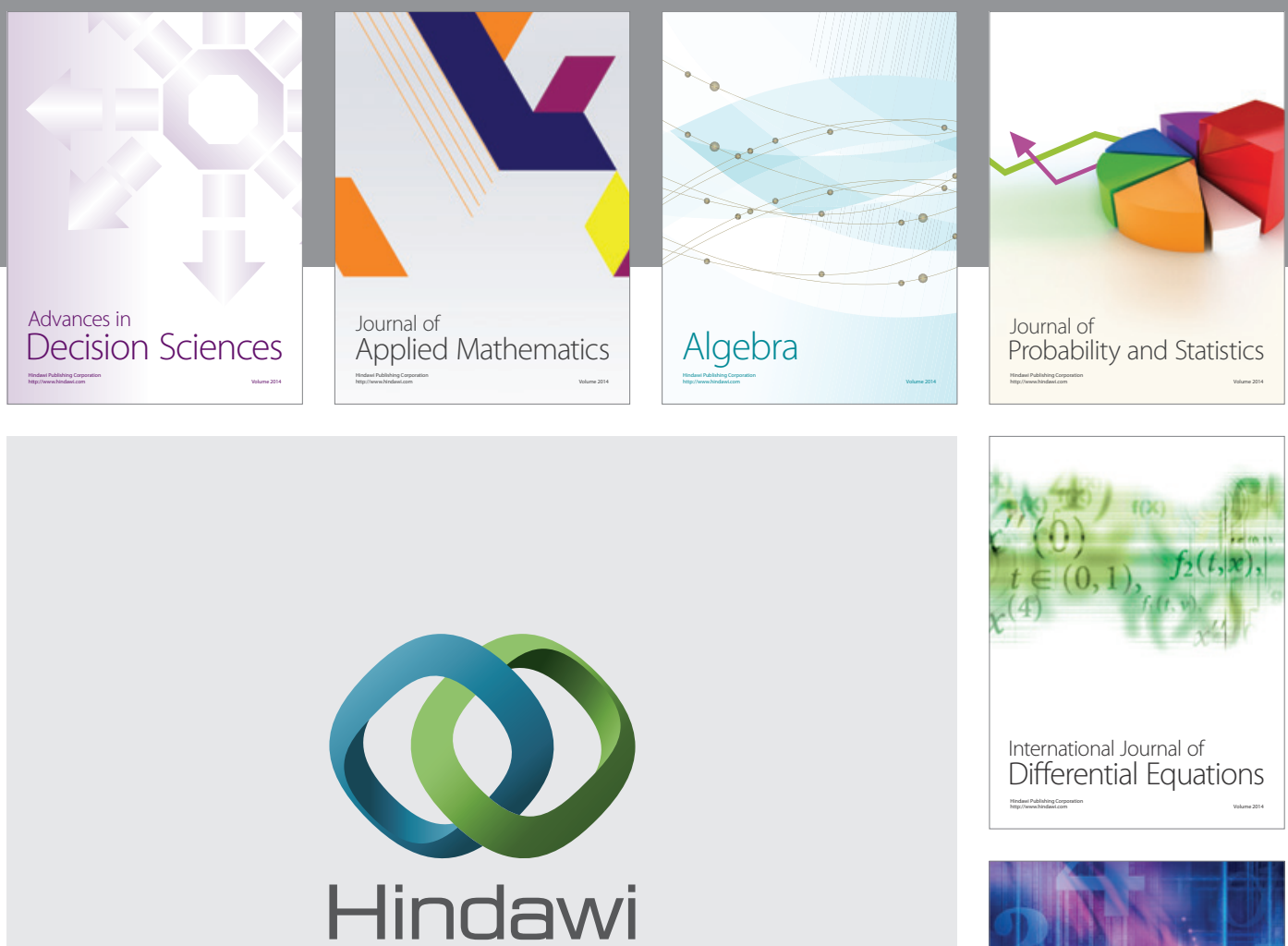

Submit your manuscripts at http://www.hindawi.com
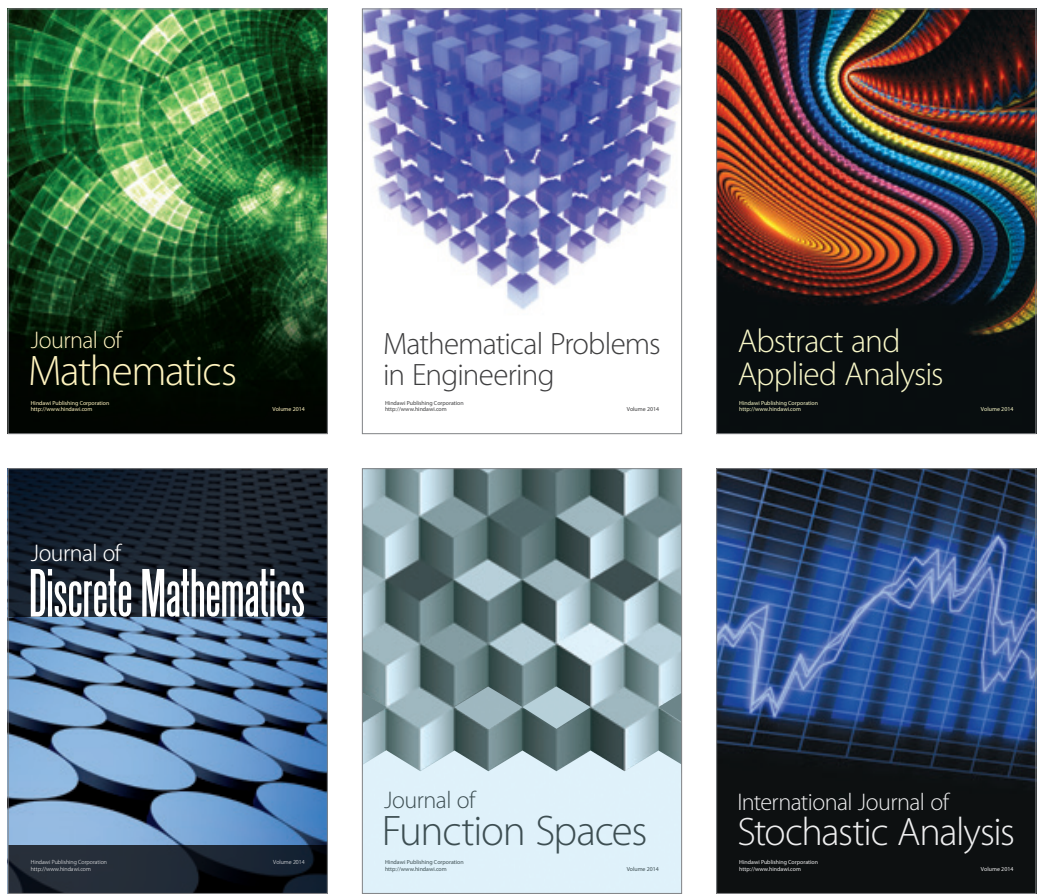

Journal of

Function Spaces

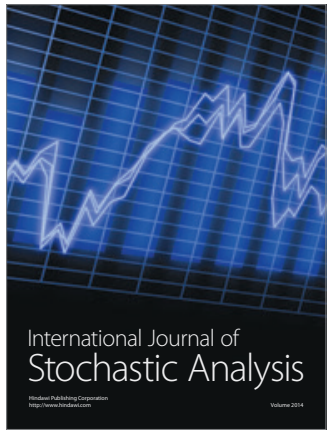

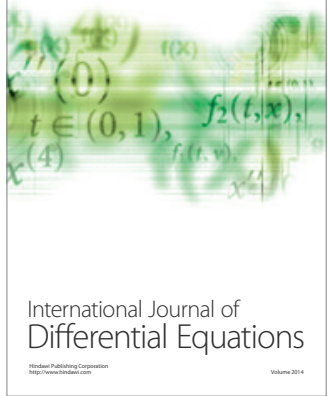
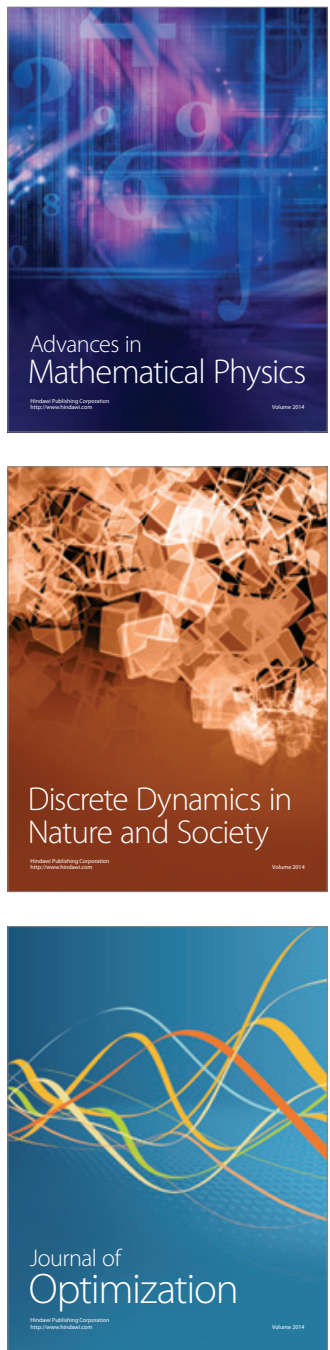